\title{
What is The $R_{0}$ Number and Clinical Significance in Infectious Diseases?
}

\section{Enfeksiyon Hastalıklarında Ro Oranı ve Klinik Anlamı Nedir?}

\author{
Cansu Turan "1](iD) \\ ${ }^{1}$ Division of Pediatric Infectious Diseases, Department of Pediatrics, Uludag University School of Medicine, Bursa, Turkey
}

Question: What is the $R_{0}$ rate and clinical significance in infectious diseases? Ceren Baylan, MD

Cite this article as: Turan C, Hacımustafaoğlu M. What is the $R_{0}$ number and clinical significance in infectious diseases? J Pediatr Inf 2020;14(1):e47-e48.

\section{Answer (Cansu Turan, MD; Mustafa Hacımustafaoğlu, MD)}

$R_{0}$ number is also known as Basic reproduction number ( $R$ zero or $\mathrm{R}$ naught in English pronunciation) and is a term used in epidemiology of infections. For an infectious disease, $R_{0}$ number shows the average number of secondary cases that develop after a typical patient (transmitted from this patient) in an entirely susceptible society. Although it is called as Basic reproductive ratio or Basic reproductive rate in some sources, it would be more accurate to call it Basic reproduction number since it indicates a certain number. The $R_{0}$ is generally denoted as a number (eg. 2.6) or numerical distribution (eg. 12-18). The main epidemiological triad variables (infection agent, host and environmental factors) are important in estimating $R_{0}$ number. Three main variables play a major role in the calculations: a) The time of infection after a person is infected, $b$ ) The possibility of contamination after contact with the susceptible person, and c) The intensity and duration of contact. Even if $R_{0}$ is specified as a single number or distribution of numbers, it can actually be influenced by a lot of epidemio- logical features of the infection (such as transmission route, incubation period, infectious period, the rate of immunity in the population), biological features of the infectious agent, socio-demographic variables (characteristics of the environment, patients and healthy individuals). So $R_{0}$ can be thought of as an epidemiological summary of many factors. Although $R_{0}$ is basically defined for a fully susceptible population for an infectious disase, it can also be estimated by some mixed calculations in cases where there is a certain level of immunity in the community.

If the $R_{0}$ ratio is greater than 1 ; it is predicted that a patient can transmit the disease to more than one person, and over time the disease will spread gradually in the community, and can cause an epidemic. If the $R_{0}$ number is less than 1 ; not every case can transmit the disease proportionally to another person, and the disease will be self-limited, over time ceased, but there may still be new some cases. Diseases with high $R_{0}$ value are more likely to spread and create epidemics in society than diseases with low $R_{0}$ value. This spreading rate can reach risky outbreaks, especially if adequate measures are not taken in the community. In practice, by saying $R_{0}=3$ for

\section{Correspondence Address / Yazışma Adresi}

Mustafa Hacımustafaoğlu

Department of Pediatrics, Department of

Pediatric Infectious Diseases,

Uludag University School of Medicine,

Bursa-Turkey

E-mail: mkemal@uludag.edu.tr 
a disease, it is understood that in a susceptible society, a sick person can transmit the disease roughly and on average to 3 healthy people.

The $R_{0}$ simply indicates how many people spread a disease in the community only from a sick person. There is no relationship between the $R_{0}$ value and the severity of the disease. Likewise, the $R_{0}$ value does not indicate how quickly the disease has spread in the community. For example, for a disease with slow incubation time, or with a long latent infection period (such as tuberculosis), appearances of secondary cases take long time even if the $R_{o}$ is high. However, some other diseases with relatively short incubation periods and without latent infections (eg measles or COVID-19) the potential risk of clinical outbreaks or epidemics will be higher.

$R_{0}$ values of different infectious diseases differ from each other. It should also be kept in mind that the $R_{0}$ value is given on average, even if it is for the same disease, it is not an absolute fixed number, and may vary according to different conditions. $R_{0}$ values are reported as on average; 6-7 in diphtheria, 5-100 in malaria, 12-18 in measles, 4-7 in mumps, 5-17 in pertussis, 5-7 in polio, 6-7 in rubella, 2-5 in SARS, 2-3 in influenza (1918 pandemic), 1.5-2 in Ebola, 0.3-0.8 in MERS and 2-5 in HIV/AIDS (with sexual contacts). In seasonal influenza, it was calculated as approximately 1.3. $R_{0}$ is generally found higher in airborne diseases. $R_{0}$ values that calculated and/or estimated for a certain disease may differ in various studies, years, social groups, etc. For example, $R_{0}$ rates calculated for measles were estimated between 3-203 (median; 6.7-15.9) in various studies conducted in the last century; in pre-vaccination and post-vaccination era, developed and developing countries, according to normal or epidemic surveillance factors, or taking into account birth rates.

When the number of immune individuals increases, the transmission/spread rate of the disease decreases and the transmission rate of the disease is interupted when it reaches a certain critical level. This condition is called as herd immunity threshold. The herd immunity threshold can be considered as the minimum vaccination coverage rate required for interrupting the spread of an infectious disease that can be protected by vaccination in the community. The herd immunity threshold for a vaccine-preventable infectious disease in regard of the vaccine coverage rate may be different for different diseases. The herd immunity threshold was estimated to be $83-94 \%$ for measles, $75-86 \%$ for mumps, $92-94 \%$ for pertussis, $80-86 \%$ for polio, $83-85 \%$ for rubella.
$R_{0}$ values have been estimated in different studies for COVID-19 disease, which has been occurring in the world and in our country for the last 3 months and warned by the World Health Organization (WHO) as a pandemic disease. The COVID-19 $R_{0}$ value was found to be between 1.4-6.49 (mean 3.28, median 2.79), by evaluating 12 studies studies published between January and February 2020, especially in China and other countries. WHO estimated it to be 1.4-2.5. In general, it can be said that the $R_{0}$ value of SARS CoV-2, which causes current COVID-19 disease, is approximately 2.6. This figure is higher than other serious coronavirus infections, such as SARS and MERS, so it can be said that COVID-19 disease has a much faster potential to spread. When we make a simple scenario, if the $R_{0}$ value was accepted as 2.6 , it was estimated that a patient with COVID-19 would infect 2.6 people after 1 transmission cycle (after 2-14 days, an average of 5 days of incubation period) and infect 368 people after 7 transmission cycles (average 35 days).

In summary, the $R_{0}$ value is a value that can reflect how many people can be infected by the index contagious case, especially for a given infectious disease that is newly emerging or the community is totally susceptible to it. However, it would be appropriate to interpret this figure rationally, by taking account together with other epidemiological factors such as with the agent of infection, host and environmental factors.

\section{References}

1. Guerra FM, Bolotin S, Lim G, Heffernan J, Deeks SL, et al. The basic reprodiction number ( $\left.R_{d}\right)$ of measles: a systematic review. Lancet Infect Dis 2017;12:e420-28. ['Cross Ref]

2. Basic reproduction number. https://en.wikipedia.org/wiki/Basic_reproduction_number. Accesed on 20.03.2020. [CrossRef]

3. Delamater PL, Street EJ, Leslie TF, Yang YT, Jacobsen KH. Complexity of the basic reproduction number $\left(R_{0}\right)$. Emerg Infect Dis 2019;25:1-4. [Crossiefi]

4. Herd immunity: history, theory, practice. Fine PEM Epidemiol Rev 1999;15:265-302. "[C CrossRef $]_{1}$

5. https://www.livescience.com/new-coronavirus-compare-with-flu. html. Accesed on 18 Mart 2020. '[C

6. Vynnycky E, White R. An introduction to infectious disease modelling. Oxford: Oxford University Press, 2010. [CrossRef]

7. https://www.medicalnewstoday.com/articals/coronavirus-may-spread-faster-than-who-estimate\#Coronavirus-spreads-faster-than-SARS.

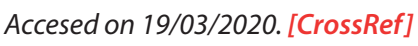

8. https://www.nytimes.com/interactive/2020/world/asia/china-coronavirus-contain.html. Accesed on 18/03/2020. [CrossRef]] 